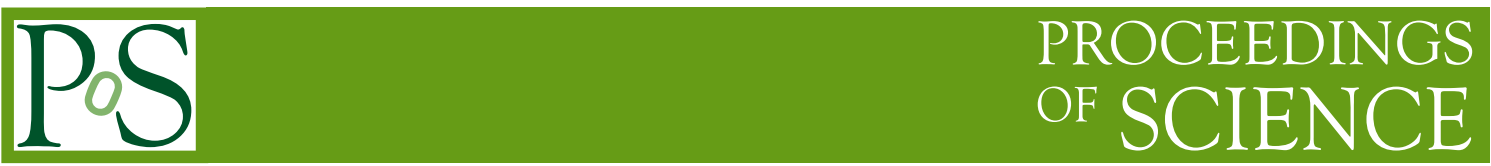

\title{
Multilayer solutions for Near-BPS Skyrme Models
}

\author{
Luc Marleau*t and Nicolas Giasson \\ Département de physique, de génie physique et d'optique, Université Laval, Canada \\ E-mail: lmarleau@phy.ulaval.ca, nicolas.giasson.1@ulaval.ca
}

The Skyrme Model is considered a natural candidate for a low-energy effective theory of QCD, a point of view supported by results coming from $1 / N_{c}$ expansion and holographic QCD. This framework leads to an attractive picture where baryons (and nuclei) emerge as topological solitons with a topological number identified to the baryon number $A$. Unfortunately, even the most naive Skyrme Model extensions have been plagued with the same problem: they predict large binding energies for the nuclei. The more recently proposed near-BPS class of Skyrme models provides a simple answer to this problem. The solutions nearly saturate the Bogomol'nyi bound which means that by construction they must have small binding energies. We present our most recent results regarding near-BPS Skyrmions and argue that they provide an improved description of nucleons and nuclei. More precisely, we address here the issue regarding the energy minimizer which remains unknown for $A>1$ by proposing a more appropriate ansatz than the usual axially symmetric solution at least for large $A$.

EPS-HEP 2017, European Physical Society conference on High

5-12 July 2017

Venice, Italy

\footnotetext{
* Speaker.

${ }^{\dagger}$ Work supported by the NSERC (Canada).
} 


\section{Introduction}

Despite the successes of the Standard Model, there remain unanswered questions among which one of the most important is due to our inability to provide a clear explanation on confinement of quarks and gluons from QCD. However it is expected that the low-energy limit of QCD should lead to an effective theory of mesons in which nucleons and nuclei emerge as topological solitons. The Skyrme Model [1] provides such a picture by establishing a direct link between baryons and soft-pion physics. In its original form, it reproduces most of the properties of the nucleon within a 30\% accuracy which is considered a rather good agreement for a model involving only two free parameters. Modifications in the structure of the potential term, the contribution of other vector mesons or simply the addition of higher-order terms in derivatives of the pion fields $[2,3,4]$ may help to improve some of the features of the model but in general these models fail to give an appropriate account of multibaryon physics or nuclei. Among the most common problems are large binding energies, shell-like baryon density configurations with unexpected discrete symmetries, as well as a nuclear radius that grows as $\sqrt{A}$ instead of the usual $|A|^{1 / 3}$ mass number dependence.

Recently, it was pointed out in refs. [5, 6, 7] that if the model was to be constructed along the lines of a BPS model it would have zero or small binding energies. A BPS soliton saturates the Bogomol'nyi bound leading to a static energy $E_{\mathrm{BPS}}(A)=E_{\mathrm{BPS}}(1)|A|$ and no binding energy which come close to what is observed experimentally. The present work is based on the more realistic extension of the original Skyrme Model called near-BPS Skyrme Model [7, 8]. The model has been shown to replicates some basic relations for nuclei: (a) a small but non zero binding energies, (b) a nuclear radius that grows $|A|^{1 / 3}$, (c) solutions that possess the symmetries of incompressible fluid and more. It is also possible to achieve a more realistic description of the baryon density configurations and binding energies per nucleon $B / A[8]$.

There remains however an open question: what is the energy minimizing solution and does it affect the nuclei properties? For the near-BPS models, lowest energy solutions are unknown for $A>$ 1 and using the usual axially symmetric (AS) ansatz [5] as an approximation leads to a potentially problematic behavior as $E_{\mathrm{nBPS}}$ grow as $A^{7 / 3}$ for large $A$. On the other hand, a complete analysis of this class of models is numerically difficult so here, in the absence of an exact energy minimizer, we aim for a simpler prospective analysis and propose to extend the usual axially symmetric ansatz to "multilayer" solutions. It turns out that distributing the energy among several layers is energically favored and modifies how nuclei masses depend on $A$ to a more acceptable behavior, i.e. $E_{\mathrm{nBPS}}$ grows roughly as $A$ for large $A$.

\section{Near-BPS Skyrme Model}

Extending the Skyrme Model [1] to a regime where the solutions become near-BPS solitons so that $M_{\text {nuclei }} \approx A \cdot M_{\text {nucleon }}$, we consider the Lagrangian density

$$
\mathscr{L}_{\text {nBPS }}=\mathscr{L}_{\text {Skyrme }}+\mathscr{L}_{\text {BPS }}
$$

Here, $\mathscr{L}_{\text {Skyrme }}=\mathscr{L}_{2}+\mathscr{L}_{4}$ is the original Skyrme Model ${ }^{1}$ consisting of the nonlinear- $\sigma$ and the Skyrme terms, respectively $\mathscr{L}_{2}=-\alpha \operatorname{Tr}\left[L_{\mu} L^{\mu}\right] \quad$ and $\quad \mathscr{L}_{4}=\beta \operatorname{Tr}\left(\left[L_{\mu}, L_{v}\right]^{2}\right)$. The pion fields

\footnotetext{
${ }^{1}$ A potential term $\mathscr{L}_{0}$ such as in eq. (2.2) is sometimes added.
} 
$\pi_{i}$ are introduced through in $U=\exp \left(-2 i(\vec{\tau} \cdot \vec{\pi}) / F_{\pi}\right)$ where $U \in S U(2), L_{\mu}=U^{\dagger} \partial_{\mu} U$ and $F_{\pi}$ is the pion decay constant. Furthermore, $\mathscr{L}_{\mathrm{BPS}}=\mathscr{L}_{0}+\mathscr{L}_{6}$ corresponds to the so-called BPS Skyrme Model proposed by Adam et al. [5,6]. It contains a potential term $V$ and the term of order six in derivative of the pion fields respectively

$$
\mathscr{L}_{0}=-\mu^{2} V(U) \quad \text { and } \quad \mathscr{L}_{6}=-\frac{3}{2} \frac{\lambda^{2}}{16^{2}} \operatorname{Tr}\left(\left[L_{\mu}, L_{v}\right]\left[L^{v}, L^{\lambda}\right]\left[L_{\lambda}, L_{\mu}\right]\right)=-\lambda^{2} \pi^{4} \mathscr{B}^{\mu} \mathscr{B}_{\mu}
$$

Here $\mathscr{B}^{\mu}=\frac{1}{24 \pi^{2}} \varepsilon^{\mu \nu \rho \sigma} \operatorname{Tr}\left(L_{v} L_{\rho} L_{\sigma}\right)$ is the baryon (or topological) current. So here, $\mathscr{L}_{6}$ has a special meaning: it is the square of the pullback of the volume form in target space. Finite energy solutions require a conserved topological charge identified with the nuclear mass number which also corresponds to the baryon number $A=\int d^{3} r \mathscr{B}^{0}$.

The BPS model energy minimizer saturates of the Bogomol'nyi bound so the static energy $E_{\mathrm{BPS}} \propto A$. Using the general form $U=\cos F+i \hat{n} \cdot \tau \sin F$ with $\hat{n}=(\sin \Theta \cos \Phi, \sin \Theta \sin \Phi, \cos \Theta)$ where $F=F(\mathbf{r}), \Theta=\Theta(\mathbf{r})$ and $\Phi=\Phi(\mathbf{r})$, ones finds an infinite number solutions with same lowest energy $E_{\mathrm{BPS}}=2 \mu \lambda \pi^{2}\langle\sqrt{V}\rangle_{S^{3}}|A|$ provided $\mu \sqrt{V}=\mp \lambda \pi^{2}\left(\sin ^{2} F \sin \Theta \nabla F \cdot(\nabla \Theta \times \nabla \Phi)\right)$. This cannot be achieved in the Skyrme Model whose minimizing energy exceeds the bound by as much as $23 \%$ leading eventually to unsatisfactory large binding energies for nuclei. On the other hand, BPS model have zero binding energies at the level of the static energy and lacks a kinetic term in $\mathscr{L}_{2}$ that would define a proper propagator.

The near-BPS Skyrme Model [7] in (2.1) slightly departs from the BPS model assuming the term $\mathscr{L}_{\mathrm{BPS}}$ dominates while treating $\mathscr{L}_{2}$ and $\mathscr{L}_{4}$ as small perturbations. The model then allows for small but non-zero binding energies as well as a kinetic term. Unfortunately, the lowest energy solutions for the near-BPS model are not known for $A>1$ and cannot be guessed from the dominant part $\mathscr{L}_{\mathrm{BPS}}$ since it possesses an infinite number of degenerate solutions. Accordingly, it is both the $\mathscr{L}_{2}$ and $\mathscr{L}_{4}$ parts that fix the shape of the energy minimizing solutions. One then usually considers an axially symmetric ansatz as in ref. [5] but in the context of the near-BPS model the energies $E_{\mathrm{nBPS}}$ grow as $A^{7 / 3}$ for large nuclei, as opposed to the linear behavior observed experimentally. Furthermore, it was shown in [9] that the solutions should instead be a so-called restricted harmonic. Yet this constraint is not sufficient to determine uniquely the lowest energy solution.

The solution proposed in this work relies on a more elaborate ansatz in the form of the $L \leq A$ concentric layers such that each layer that corresponds to a segment of length $\pi$ in $F(r)$ winds up $m_{l}$ times around the symmetry axis. The solution can be written as $U=\cos F(r)+i \hat{\mathbf{n}}_{l}$. $\tau \sin F(r)$ for $0 \leq F \leq L \pi$ with

$$
\hat{\mathbf{n}}_{l}=\left(\sin \theta \cos m_{l} \varphi, \sin \theta \sin m_{l} \varphi, \cos \theta\right)
$$

thereby carrying a topological charge $m_{l}$ for layer $l$ such that $A=\sum_{l=1}^{L} m_{l}$. Allowing for multilayer solutions, $L \geq 1$, the total static energy get a contribution of the each Lagrangians $\mathscr{L}_{i}$ in eq. (2.1) according to

$$
E_{\mathrm{nBPS}}=\sum_{l=1}^{L}\left[\left(a_{0}^{l}+a_{6}^{l}\right) m_{l}+m_{l}^{1 / 3}\left(a_{2}^{l}+b_{2}^{l} m_{l}^{2}\right)+m_{l}^{-1 / 3}\left(a_{4}^{l}+b_{4}^{l} m_{l}^{2}\right)\right]
$$

where the model dependent quantities $a_{i}^{l}, b_{i}^{l}$ are computed for to each part of the Lagrangian $\mathscr{L}_{i}$ and layer $l$. It then remains to find which configuration of layer winding numbers $\left(m_{1}, m_{2}, \ldots, m_{L}\right)$ has 
the lowest energy $E_{2}+E_{4}$ since all layers contribute equally to $E_{0}$ and $E_{6}$. The axially symmetric ansatz in [5] corresponds to the 1-layer case ( $L=1, m_{1}=A$ in (2.4)) and generates terms proportional to $A^{7 / 3}$ and $A^{5 / 3}$ which are problematic for large nuclei. It turns out that the distribution of the topological charge over several layers attenuates the large $A$ behavior.

It should be noted that in order that the solution (2.3) remains a minimizing solution of $\mathscr{L}_{\mathrm{BPS}}$, it must saturate the Bogomol'nyi bound. This requires that $\langle\sqrt{V}\rangle_{l}$ be the same for all layers. Taking this into account, we proposed three simple multilayer near-BPS models using $\beta=0$ : (a) Model 1 (constant potential): $V_{\mathrm{M} 1}(F)=1$, (b) Model 2 (oscillating potential): $V_{\mathrm{M} 2}(F)=\sin ^{4} F$ and (c) Model 3 (constant potential dropping near surface): $V_{\mathrm{M} 3}(F)=1$ for $F \geq F_{0}$ and $V_{\mathrm{M} 3}(F)=\frac{\sin ^{4} F}{\sin ^{4} F_{0}}$ for $0 \leq F \leq F_{0}$ where $F_{0}$ is chosen so that $\langle\sqrt{V}\rangle_{l}$ are all equal.

The apparently simple form of these models however hides a technical difficulty. When the model allows for zeros in the quantity $\frac{\sin ^{2} F}{\sqrt{2 V(F)}}$, the BPS differential equation for $F$ causes $F^{\prime}$ and $E_{2}$ to diverge (for example here $V_{\mathrm{M} 1}$ and $V_{\mathrm{M} 3}$ at $F=n \pi$ with $n \in \mathbb{N}^{*}$ ). In such cases, one cannot neglect $\mathscr{L}_{2}$ and $F$ can be obtained from $F^{\prime}(x)=-\left[\frac{1}{V}\left(\left(\frac{m_{l}}{24 A} \frac{\sin ^{2} F}{x^{2}}\right)^{2}+A^{2 / 3} \gamma^{2}\right)\right]^{-1 / 2}$ where $x=a r$ with $a=(\mu / 18 A \lambda)^{2 / 3}$ and $\gamma=\sqrt{2 \alpha}\left(18 \lambda \mu^{2}\right)^{-1 / 3}$. Otherwise the BPS solution (the solution with $\gamma=0$ ) exists for all $F$ and can be used to estimate $E_{2}$. But for such models ( $V_{\mathrm{M} 2}$ for example), the baryon density must be zero at the frontier of each layer where $F=n \pi$ thereby forming distinct concentric shells in the baryon density.

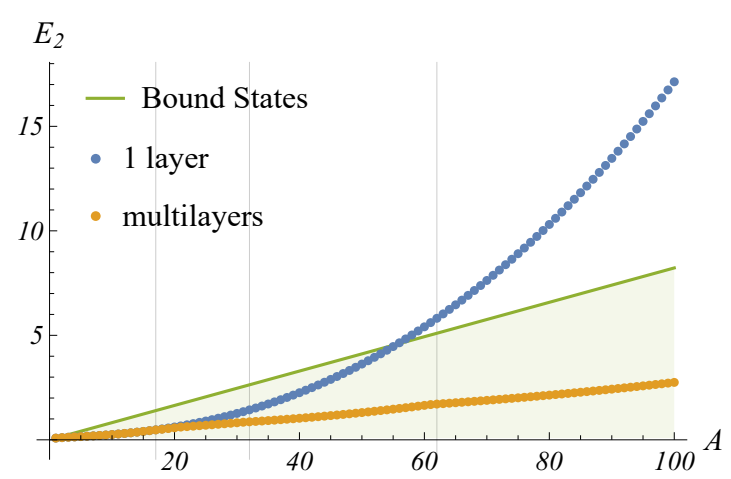

(a)

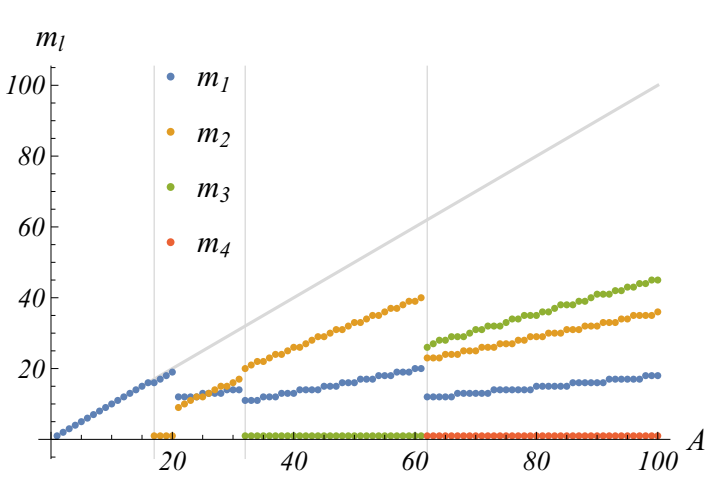

(b)

Figure 1: Model 1: (a) Static energy $E_{2}$ (in units of $\mu \lambda$ ) for single and multilayer configurations. Configurations lying in the shaded area are bound states. (b) Distribution of $m_{l}$ for energy minimizer. The vertical gray lines indicates transitions in the number of layers.

The search for a minimizing configuration requires that we set the value of $\gamma$ whereas the parameters $\mu$ and $\lambda$ may be use to rescale the energy and radial distance in units of $\mu \lambda$ and $a$ respectively. In Fig.1, we present our results for Model 1 using $\gamma=0.001$, which is typical of the values obtained in previous fit for near-BPS calculations [7]. The static energy $E_{2}$ is compared for single and multilayer configurations in Fig.1(a). All configurations in the shaded area are lower in energy than $A$ infinitely separated skyrmions and can be considered as bound states. We find that, not only the multilayer configurations are energy favored for large $A$, but $E_{2}$ seems to grow almost linearly with $A$ in that limit. This behavior is certainly more in agreement with experiment than the 1-layer results. Furthermore, Fig.1(b) illustrates how the baryon number is distributed 
among layers (layer 1 is the innermost layer). It turns out that for all models, the number of layers increases rather slowly with $A$ and one also observe some regularity on how $A$ is distributed among layers. However, this exact pattern of distribution seems to be model dependent. For example in Model 1 (see Fig. 1(b)), the baryon number carried by each layer $m_{l}$ generally increases as it gets further from the center except for the outermost layer which carry the lowest charge. Model 2 and Model 3 however follow completely different patterns yet multilayers are still energically favored.

\section{Conclusion}

In this work, we proposed a multilayer axially symmetric ansatz for the near-BPS Skyrme model. Our calculations show that multilayer configurations are energically favored as the baryon number $A$ increases and that they correspond to bound states. This should remain true for even larger $A$, at least for the prototype models at hand, since their static energy $E_{\mathrm{nBPS}}$ only grows as $\sim$ $A$. So, although the exact energy minimizer remains unknown for $A>1$, we can assume that nearBPS Skyrmions are bound states as well. Further analysis is required to investigate the cases with $\beta \neq 0$, compute rotational and Coulomb contributions to the nuclear masses and verify if the nearBPS models successes (such as the results for $B / A$ shown in [8]) are preserved. More generally, these results support the idea that nuclei could be topological solitons emerging from an effective field theory of mesons, perhaps as near-BPS Skyrmions.

\section{References}

[1] T. Skyrme, Particle states of a quantized meson field, Proc.Roy.Soc.Lond. A262 (1961) 237-245.

[2] L. Marleau, Modifying the Skyrme model: Pion mass and higher derivatives, Phys.Rev. D43 (1991) 885-890.

[3] B. Dion, L. Marleau and G. Simon, Skyrmions from a Born-Infeld action, Phys.Rev. D53 (1996) 1542-1547, [hep-ph/9408403].

[4] L. Marleau and J. F. Rivard, A generating function for all orders skyrmions, Phys. Rev. D63 (2001) 036007, [hep-ph/0011052].

[5] C. Adam, J. Sanchez-Guillen and A. Wereszczynski, A Skyrme-type proposal for baryonic matter, Phys.Lett. B691 (2010) 105-110, [1001.4544].

[6] C. Adam, C. Naya, J. Sanchez-Guillen, R. Vazquez and A. Wereszczynski, The Skyrme model in the BPS limit, 1511.05160.

[7] E. Bonenfant, L. Harbour and L. Marleau, Near-BPS Skyrmions: Non-shell configurations and Coulomb effects, Phys. Rev. D85 (2012) 114045, [1205.1414].

[8] M.-O. Beaudoin and L. Marleau, Near-BPS Skyrmions: Constant baryon density, Nucl. Phys. $\mathbf{B 8 8 3}$ (2014) 328-349, [1305.4944].

[9] J. Speight, Near BPS Skyrmions and Restricted Harmonic Maps, 1406.0739. 